RUNNING HEAD: WEAKENED RESILIENCE DURING QUARANTINE

Resilience of Adolescents, Though Weakened During Pandemic-Related Quarantine, Serves as a Protective Process Against Depression and Sleep Problems Huangqi Jiang ${ }^{1}$, Wenle $\mathrm{Yu}^{2}$, Danhua $\mathrm{Lin}^{3}$, and Brooke N. Macnamara ${ }^{1}$

${ }^{1}$ Department of Psychological Sciences, Case Western Reserve University, OH, USA ${ }^{2}$ Department of Psychology, Tsinghua University, Beijing, China

${ }^{3}$ Institute of Developmental Psychology, Beijing Normal University, Beijing, China Author Note

Huangqi Jiang https://orcid.org/0000-0001-6814-5810

Brooke N. Macnamara https://orcid.org/0000-0003-1056-4996

Correspondence should be addressed to Danhua Lin (Beijing Normal University, Beijing, China, 100875, Email: danhualin@bnu.edu.cn). 


\begin{abstract}
Adolescents facing adversities are susceptible to depression and sleep problems. Resilience is an important protective mechanism for coping with difficulties. During the COVID-19 pandemic, adolescents faced multiple hardships including being pulled from their schools and being unable to socialize with friends. Yet, it is unclear whether adolescents' resiliency weakened during the global crisis or if the protective power of resilience is maintained under such circumstances. Here, in a partially longitudinal study, we demonstrate that Chinese adolescents' resilience weakened during the pandemic-related quarantine compared to before the pandemic. However, resilience protected against depression and sleep problems for adolescents. A mediation model showed that higher resilience was associated with fewer depressive symptoms, which in turn reduced sleep problems. Moreover, social support moderated this mediation. Our findings suggest that, though the resilience of adolescents weakened during the pandemic-related quarantine, it still serves as a protective process helping adolescents cope with adversities such as depression and sleep problems. Implications for clinical practice and future research are discussed.
\end{abstract}

Keywords: resilience, adolescents, quarantine, depression, sleep problems, social support 


\section{Resilience of Adolescents, Though Weakened During Pandemic-Related Quarantine, Serves as a Protective Process Against Depression and Sleep Problems}

Quarantine during the COVID-19 pandemic was implemented as an effective way to control the transmission of COVID-19. However, quarantine for adolescents and children may cause potential problems for their mental health. In general, social isolation in adolescents is associated with higher risk of depressive symptoms, suicide attempts, and low self-esteem (Hall-Lande et al. 2007). These negative outcomes also occur when the isolation is due to disease-related quarantine. For example, during the Middle East Respiratory Syndrome (MERS) outbreak in Korea, people reported more anxiety symptoms and feelings of anger during isolation than after the release from isolation, even among people who never became infected (Jeong et al. 2016). A recent review (Brooks et al. 2020) found that most studies examining psychological effects during past quarantines reported negative mental health outcomes including confusion, anger, and post-traumatic stress symptoms. Posttraumatic stress syndrome is particularly prevalent among children in quarantine (Brooks et al. 2020), with thirty percent of children who experience disease-related quarantine and isolation meeting the criteria of posttraumatic stress disorder (Sprang and Silman 2013).

The emerging research on mental health during COVID-19-related quarantines is mirroring the findings from past disease-related quarantines. In Colombia, individuals experiencing higher stress related to the COVID-19 lockdown are at a significantly higher risk of committing suicide (Caballero-Domínguez et al. 2020). In particular, adolescents’ mental health has been negatively impacted by COVID-19 pandemic quarantine, with reports 
demonstrating high rates of negative coping strategies, post-traumatic stress disorder, depression, and sleeping problems (Li et al. 2020; Liang et al. 2020; Zhou et al. 2020).

What might buffer the negative consequences of isolation on adolescents' mental health? Resilience is a candidate factor. Resilience is an individual's ability to tolerate adversity. Resilience protects adolescents' mental health when facing difficult circumstances (Levesque 2011; Quinton et al. 1993; Zolkoski and Bullock 2012), including during and following trauma (e.g., Hébert et al. 2014; Ying et al. 2014). Numerous studies have demonstrated positive correlations between resilience and indicators of mental health (e.g., happiness, self-esteem), and negative correlations between resilience and indicators of mental health problems (e.g., anxiety, depression) among adolescents (e.g., Hjemdal et al. 2011; Mota and Matos 2015; Ziaian et al. 2012). We sought to understand the role resilience plays to protect adolescents' mental health during a pandemic-related quarantine.

First, we asked whether adolescents' resilience was impacted by pandemic-related quarantine. Some research has suggested that adolescents' resilience can be altered. For example, a school-based multiple-strategic intervention was able to improve students' resilience and protective factors one year after the three-year intervention (Hodder et al. 2011). Likewise, a recent resilience-based intervention successfully improved resilience and related psychological well-being among migrant Chinese adolescents (Tam et al. 2020). However, there is a lack of evidence about whether adolescents' resilience might fluctuate during a pandemic-related quarantine, relative to before the pandemic. 
Second, we sought to examine the role of resilience as a protective factor. According to the protective processes model, resilience can reduce the likelihood of negative chain reactions caused by the risk event (Rutter 1987). Specifically, previous research suggests that resilience protects adolescents from suffering from a range of adversities (e.g., Ye et al. 2016; Zhou et al. 2016), including depressive symptoms and sleep problems (Chatburn et al. 2014; Hjemdal et al. 2011). Recent research during the COVID-19 pandemic demonstrated that adolescents suffered from depression and sleep problems (Li et al. 2020; Zhou et al. 2020). Thus, we specifically ask whether resilience is able to serve as a protective process against depression and sleep problems during pandemic-related isolation.

More specifically, previous research has demonstrated that depressive symptoms predict the development and persistence of sleep problems in adolescents (Patten et al. 2000). In addition, adolescents who were diagnosed as major depressive disorder were found more likely to suffer from the insomnia in the 12-month follow-up than those who were not diagnosed as major depressive disorder, and insomnia was related most strongly to major depressive disorder comparing to other psychiatric disorders (Ford and Kamerow 1989). Thus, depressive symptoms may predict sleep problems and, furthermore, play a mediating role in the relationship between resilience and sleep problems.

External resources should also be considered in terms of adolescents' mental health during the pandemic-related quarantine. Social support is an important external factor potentially affecting the relationships among resilience, depression, and sleep problems. Cohen and Wills (1985) proposed that social support benefits psychological outcomes by 
serving as a buffer against impacts from adversity, especially when individuals are under stress. The buffer model is often used to account for the development and maintenance of depression (Pössel et al. 2018). For example, community violence exposure is associated with depressive symptoms of adolescents with low social support, but not among adolescents with high social support (Kaynak et al. 2011). As another example, social support from parents has been shown to moderate the relationship between negative life events and depressive symptoms in adolescents (Murberg and Bru 2004). Furthermore, social support has been found to increase sleep quality and moderate the effects of academic stress on sleep in adolescents (van Schalkwijk et al. 2015). Thus, both resilience, an internal resource, and social support, an external resource may be important factors for buffering the negative effects of pandemic-related quarantine. Specifically, social support may moderate one or more of the relationships among resilience, depression, and sleep problems.

\section{Current Study}

Our research questions, hypotheses, and analysis plan were pre-registered on the Open Science Framework (https://osf.io/gfntd). Our dataset contains one longitudinal measure — resiliency — administered before and during the COVID-19 pandemic. We further examined relationships among measures administered during the COVID-19 pandemic: depressive symptoms, sleep, and social support. In our analyses, we seek to answer four main research questions.

First, we ask how resilience among adolescents differs during a pandemic compared to before the pandemic. Second, we ask whether the resilience of adolescents can predict 
depressive symptoms and sleep problems during a pandemic when all adolescents are facing pandemic-related challenges. While resilience generally negatively predicts depressive symptoms and sleep problems under normal circumstances, it is possible that this relationship changes under the unique circumstances of a pandemic requiring social isolation.

Third, we ask whether depression mediates the relationship between resilience and sleep problems. Finally, contingent on whether depression serves as a mediator between resilience and sleep problems, we ask whether social support moderates the mediation model. According to Cohen and Wills (1985), social support has both direct and buffering effects on negative health and well-being outcomes, therefore, social support may moderate the relationships among resilience, depression, and sleep problems.

\section{Materials and Methods}

\section{Participants}

The sample consisted of 257 middle-school students in southern China. The students all attended the same school and lived in middle-class neighborhoods. Data were collected during the pandemic when all students in the sample were attending school from home and were engaging in mandatory preventative social distancing. The city has a population of 4 million. At the time of data collection, there were only 35 confirmed cases in the city $(<$ $0.00001 \%$ ) and zero COVID-19-related deaths ${ }^{1}$. Thus, the majority of stress during this time

\footnotetext{
${ }^{1}$ Information from the Municipal Health Commission. Retrieved April 21st, 2020.
} <http://hfpc.xm.gov.cn/xwzx/tzgg/202004/t20200421_2440663.htm> 
was likely due to isolation more so than fearing for one's own wellbeing or the wellbeing of friends and family.

The mean age of participants at the time of measurement was $13.85(S D=1.29)$ years, and the age range was 11-16 years. Of the 257 participants, 124 were female and 133 were male. Online informed consent was obtained from participants and their parents prior to the initiation of the investigation. The participants were asked to independently complete the online questionnaire without help from their parents.

Of the 257 participants, 188 participants' resilience were measured before the pandemic in an in-school survey. Permission to use the measure of resilience before the pandemic in this study was granted from participants during the collection of the other measures during the pandemic.

\section{Procedure}

Resilience before the pandemic was measured between November $29^{\text {th }}, 2019$ and December $9^{\text {th }}, 2019$. The data collection amid the pandemic started on April $9^{\text {th }}, 2020$, and ended on April 17 $7^{\text {th }}, 2020$. During this time, the participants' school was closed, students were taking remote classes at home and were required to continue social distancing practices.

The study was approved by the Institutional Review Board at Department of Psychology of Tsinghua University. The purpose of the study and the voluntary nature of the students' participation were highlighted before the questionnaire. After the online questionnaire, participants were told that school psychologists or teachers were available to provide counseling services if needed. 


\section{Measures}

The data analyzed in this report is a subset of a larger dataset collected before and during the pandemic. All measures collected during the pandemic were pre-registered on the Open Science Framework (https://osf.io/ctudz). The results of other parts of the dataset are to be reported elsewhere.

\section{Resilience}

Resilience was measured by the Resilience Scale for Chinese Adolescents (RSCA). It is a 27-item, self-rating measure (Hu and Gan 2008). Each item was rated on a 5-point Likert scale $(1=$ completely disagree , and $5=$ completely agree $)$. An example item is "I am always discouraged by failure.” The measure consists of five components: goal planning, help seeking, family support, affect control, and positive thinking. Goal planning addresses individuals' propensity to adhere to goals, make plans, and focus on solving problems that interfere with these goals. Help seeking addresses individuals' propensity to find people with whom they can talk about their problems.. Family support addresses an individual's perception of respect, encouragement, and support from family members. Affect control addresses individuals' ability to moderate their emotions when facing failure and other adversities. Positive thinking addresses individuals' propensity to focus on the potential benefits from facing adversities. The total score was the sum of all items. Higher scores represent higher levels of resilience. In this study, the internal reliabilities both before and during the pandemic were excellent: both $\alpha \mathrm{s}=.90$. 


\section{Depression}

Adolescents' depressive symptoms were measured using the Center for Epidemiologic Studies Depression Scale for Children (CES-DC) (Fendrich et al. 1990). The CES-DC consists of 20 standardized items for the assessment of emotional, cognitive, and behavior-related symptoms of depression. For each item, participants are instructed to assess the frequency of their reactions in the past week. An example item is "I was bothered by things that usually don't bother me.” All items are evaluated with 4-point response options (0 $=$ not at all, $1=$ a little, $2=$ some, $3=$ a lot . . Total possible scores range from 0 to 60 . Higher CES-DC scores represent increased levels of depressive symptoms. Fifteen points and above may be the cutoff for screening children and adolescents for major depressive disorder (Fendrich et al., 1990). Good reliability and construct validity have been found among Chinese population (William Li et al. 2010). In this study, the internal reliability was excellent $(\alpha=.93)$.

\section{Sleep problems}

Sleep problems were measured by the Pittsburg Sleep Quality Index (PSQI), which asked participants to report sleep habits in the past month (Buysse et al. 1989). It consists of seven components of sleep quality: subjective sleep quality, sleep latency, sleep duration, habitual sleep efficiency, sleep disturbances, use of sleep medication, and daytime dysfunction. The global sleep quality index was the sum of these seven components, ranging from 0 to 21, with higher scores representing more severe sleep problems. An example item is "During the past month, how often have you had trouble sleeping because you have to get 
up to use the bathroom?" A global sleep quality index greater than 5 indicates "poor" sleep quality (Buysse et al. 1989). In this study, the internal reliability of the seven components was acceptable $(\alpha=.74)$.

\section{Social support}

Social support was measured by the Multidimensional Scale of Perceived Social Support Scale (MSPSS), which asked participants to respond to each question on a 7-point Likert scale $(1=$ very strongly disagree , and 7 = very strongly agree $)($ Zimet, Dahlem, Zimet, \& Farley, 1988). An example item is "I have friends with whom I can share joys and sorrows." The MSPSS measures three sources of social support: friends, families and significant others. The Chinese version of the MSPSS has shown good reliability and validity among Chinese adolescents (Zhao et al. 2018). In this study, the internal reliability was excellent $(\alpha=.94)$.

\section{Data Analysis}

Pearson correlations and paired-samples $t$-tests were conducted to check the consistency of resilience before and during the pandemic. We also report Bayes Factor (BF) in support of the alternative hypotheses using JASP (JASP Team 2019). Descriptive analyses and correlations of study variables were conducted. All statistical analyses were conducted using SPSS24. Statistical significance was defined as a two-tailed $p$-value of $<.05$. All models were controlled for covariates of age and gender and the predictors were centered on the mean. 
The mediation and moderated mediation models were analyzed using the PROCESS macro for SPSS (Hayes 2013). The bias-corrected 95\% confidence interval (CI) was calculated with 10,000 bootstrap samples. First, we tested whether the association between resilience and sleep problems was mediated by depressive symptoms (see left panel of Fig. 1). If the $95 \%$ confidence interval of the indirect effect did not contain 0 , it indicated that the mediating effect was significant. Next, we examined moderated mediation, that is, whether social support moderated the direct or indirect effects of resilience on sleep problems (see right panel of Fig. 1). If the $95 \%$ confidence interval of the interaction did not contain 0 , a significant moderated mediation effect could be established.

Fig. 1

Schematic models

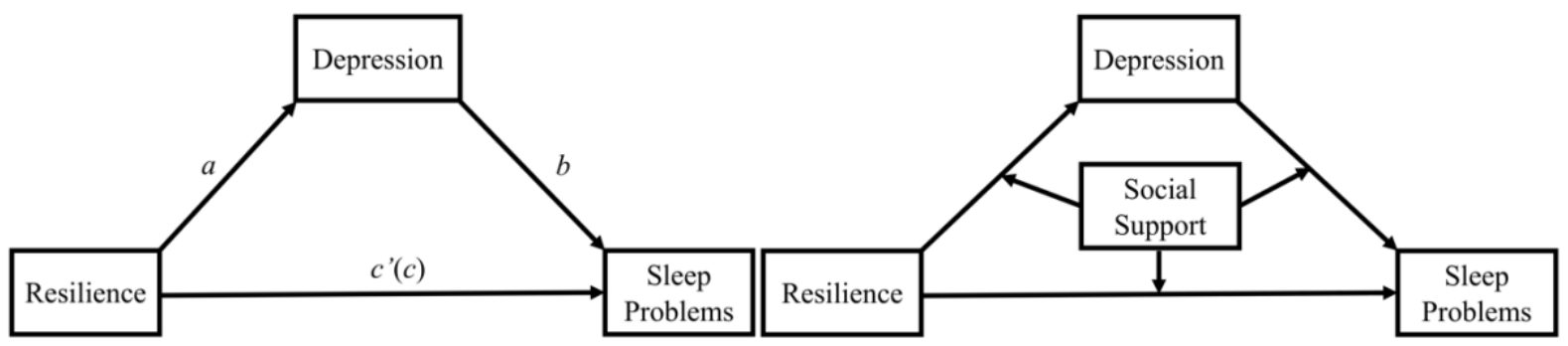

Note. Left panel: schematic model of depression as the mediator between resilience and sleep problems (Model 4 in PROCESS). Right panel: schematic model of social support as a moderator of the mediation model (Model 59 in PROCESS) 


\section{Results}

\section{Correlation of Resilience Scores Before and During the Pandemic}

Resilience scores before and during the pandemic were correlated, $r(188)=.46 p$

$<.001$. While the results align with our hypothesis that the resilience scores before and during the pandemic would be positively correlated, the moderate strength of the correlation suggests the reliability of the measure was influenced by the pandemic-related quarantine.

\section{Differences in Resilience Before and During the Pandemic}

A paired-samples $t$-test was conducted to test the difference in resilience before and during the pandemic. In line with our hypothesis, we found that resilience during the pandemic $(M=93.12, S D=10.37)$ was lower than before the pandemic, $M=100.26, S D=$ 17.68, $t(187)=-6.15, p<.001$, Cohen's $d=-0.45$. See Fig. 2 .

\section{Fig. 2}

Resilience of adolescents before and during the pandemic.

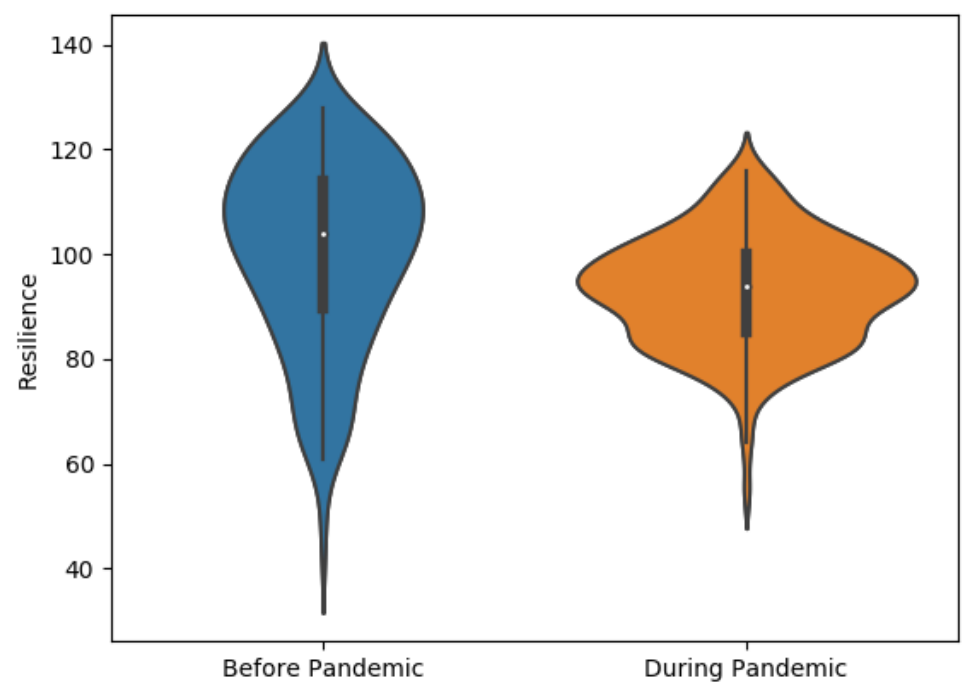

Note. The resilience during the pandemic was lower than before the pandemic 
We explored the change of each component of the RSCA. With the exception of the family support component, which slightly increased during the pandemic compared to before the pandemic, $t(187)=2.61, p=.010$, Cohen's $d=0.19$, all other components of resilience decreased significantly. See Table 1.

Table 1

Comparisons for components and total of resilience before and during the pandemic

\begin{tabular}{lccccc}
\hline & $\begin{array}{c}\text { Before } \\
\text { pandemic }\end{array}$ & $\begin{array}{c}\text { During } \\
\text { pandemic }\end{array}$ & $t$ & Cohen's $d$ & $\begin{array}{c}\text { Bayes } \\
\text { factor }\end{array}$ \\
\hline Goal planning & $19.95 \pm 4.48$ & $18.52 \pm 3.73$ & $-4.35 * * *$ & -0.32 & 589.95 \\
Help seeking & $22.31 \pm 6.38$ & $18.90 \pm 2.70$ & $-7.64 * * *$ & -0.56 & $>10^{9}$ \\
Family support & $19.44 \pm 2.88$ & $20.18 \pm 3.53$ & $2.61 *$ & 0.19 & 2.18 \\
Affect control & $21.97 \pm 6.19$ & $19.80 \pm 2.74$ & $-4.88^{* * *}$ & -0.36 & 5175.15 \\
Positive thinking & $16.58 \pm 3.69$ & $15.72 \pm 2.68$ & $-3.31 * *$ & -0.24 & 15.41 \\
Total resilience & $100.26 \pm 17.68$ & $93.12 \pm 10.37$ & $-6.15^{* * *}$ & -0.45 & $>10^{6}$ \\
$* p<.05, * * p<.01, * * * p<.001$. & & &
\end{tabular}

\section{Descriptive Statistics and Correlations for Measures Administered During the}

\section{Pandemic}

Descriptive statistics and the correlations among the various measures administered during the pandemic are shown in Table 2. We did not find gender difference on any measure. With the exception of age, all measures were significantly correlated with one another. Aligning with our hypotheses, the resilience of adolescents during the pandemic was associated with depressive symptoms and sleep problems.

Of the 188 participants who provided pre- and during pandemic measures of resilience, 18 were excluded due to missing scores on the PSQI scale. We also explored 
whether resilience measured before the pandemic rather than the measure during the pandemic-related quarantine yielded similar correlations between measures administered during the pandemic.

Table 2

Means and standard deviations for and correlations between age, resilience, depression, sleep problems, and social support

\begin{tabular}{lcccccc}
\hline & Mean \pm SD & 1 & 2 & 3 & 4 & 5 \\
\hline (1) age & $13.85 \pm 1.29$ & -- & -.13 & .08 & -.05 & -.07 \\
(2) resilience & $92.49 \pm 10.33$ & -.09 & -- & $-.37 * * *$ & $-.18^{*}$ & $.36^{* * *}$ \\
(3) depression & $15.34 \pm 11.59$ & .09 & $-.55^{* * *}$ & -- & $.65^{* * *}$ & $-.50^{* * *}$ \\
(4) sleep problems & $5.01 \pm 3.32$ & .03 & $-.31 * * *$ & $.68^{* * *}$ & -- & $-.32^{* * *}$ \\
(5) social support & $63.23 \pm 14.47$ & -.04 & $.69 * * *$ & $-.60^{* * *}$ & $-.41 * * *$ & -- \\
\hline
\end{tabular}

Note. Correlations between resilience and other measures during the pandemic-related quarantine are shown below the diagonal. Correlations between resilience before the pandemic and other measures during the pandemic-related quarantine are shown above the diagonal. Means and standard deviations for resilience are those measured during the pandemic.

$* p<.05, * * * p<.001$.

\section{Mediation Analyses}

The results of the mediation analysis demonstrated that the total effect of resilience on sleep problems was significant $(B=-.10, p<.001)$. The significant coefficient of path $a(B=$ $-.61, p<.001)$ and path $b(B=.21, p<.001)$ indicated a negative association between resilience and depressive symptoms, and a positive association between depressive symptoms and sleep problems. Further, the point estimate of the indirect effect (path $a \times b$ ) between 
resilience and sleep problems through depression was -.13 $(S E=.02)$, and the $95 \%$ biasedcorrected bootstrap confidence interval was -.16 to -.10 , which indicated that the indirect effect of resilience on sleep problems was statistically significant. In addition, the direct effect of resilience on sleep problems after including depressive symptoms as a mediator was no longer significant (path $c^{\prime}=-.03, p=.109,95 \% \mathrm{CI}=[-.01, .06]$ ), indicating that depressive symptoms fully mediated the relationship between resilience and sleep problems.

We also explored whether resilience measured before the pandemic rather than the measure during the pandemic-related quarantine yielded similar results. We found that prepandemic resilience also significantly predicted depressive symptoms and sleep problems during the pandemic-related quarantine $(B=-.25, p<.001$, and $B=-.04, p=.042$, respectively). When we conducted the mediation model with the pre-pandemic measure of resilience, we found that the pattern of the mediating effect of depressive symptoms held, though with a weaker effect $(B=-.05, S E=.01,95 \% \mathrm{CI}=[-.07,-.03])$. See Table $\mathrm{S} 1$.

\section{Moderated Mediation Analyses}

According to our hypotheses, social support may function as a moderator between resilience and sleep problems either directly or by interacting with another path in the model. Social support moderated the effect of resilience during the pandemic on depressive symptoms, $B=.01, p=.001,95 \% \mathrm{CI}=[.004, .02]$. However, social support did not moderate the relationship between resilience and sleep problems, $B=-.001, p=.550,95 \% \mathrm{CI}=$ $[-.003, .001]$, or the relationship between depressive symptoms and sleep problems, $B=.002$, $p=.086,95 \% \mathrm{CI}=[-.000, .003])$. Table 3 and Fig. 3 show the results of the moderated 
mediation model with the two null paths removed. See Table S2 for the full moderated mediation model.

Table 3

The results of the moderated mediation model analyses

\begin{tabular}{|c|c|c|c|c|c|c|c|c|}
\hline \multicolumn{2}{|c|}{ Variables } & \multicolumn{3}{|c|}{ Overall Model Fit } & \multicolumn{4}{|c|}{ Significance of Regression Coefficient } \\
\hline Outcome & Predictor & $R$ & $R^{2}$ & $F$ & $B$ & LLCI & ULCI & $t$ \\
\hline \multirow[t]{4}{*}{ Depression } & Resilience & .65 & .42 & $36.79 * * *$ & -.26 & -.41 & -.12 & $-3.53 * * *$ \\
\hline & Social support & & & & -.32 & -.43 & -.22 & $-6.02 * * *$ \\
\hline & Resilience $\times$ Social & & & & .01 & .004 & .02 & $3.30 * *$ \\
\hline & support & & & & & & & \\
\hline \multirow[t]{2}{*}{ Sleep problems } & Resilience & .68 & .47 & $55.03 * * *$ & .03 & -.01 & .06 & 1.61 \\
\hline & Depression & & & & .21 & .18 & .24 & $13.21 * * *$ \\
\hline
\end{tabular}

Note. LLCI, lower level confidence interval. ULCI, upper level confidence interval. Age and gender were controlled in the mediation analyses.

$* * p<.01, * * * p<.001$ 
Fig. 3

Moderated mediation model among resilience, depression, social support, and sleep problems

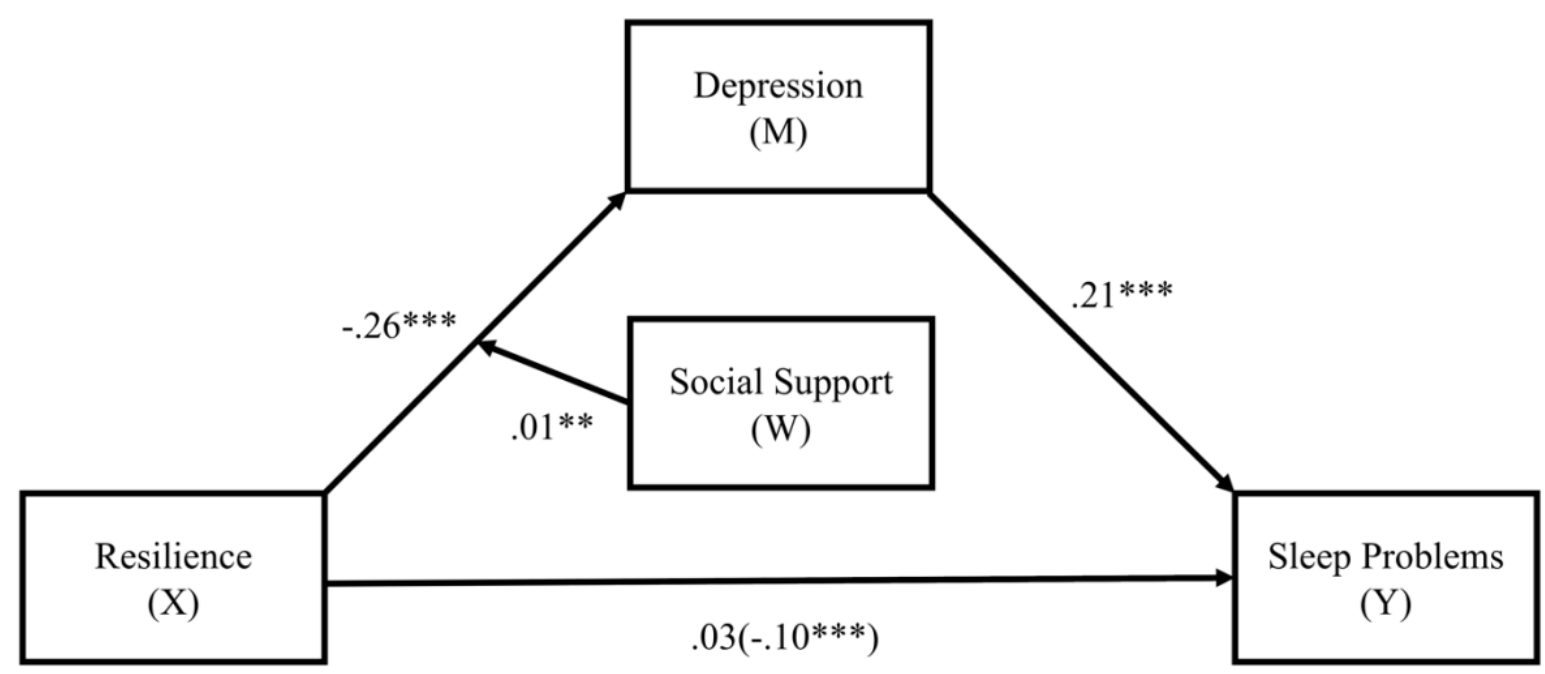

$* * p<.01, * * * p<.001$

Fig. 4 reflects how the influence of resilience on depressive symptoms was moderated by social support. When the degree of social support was low, resilience negatively predicted depressive symptoms, whereas when the degree of social support was high, depression did not differ across different degrees of resilience. 
Fig. 4

The moderating effect of social support on the relationship between resilience and depression

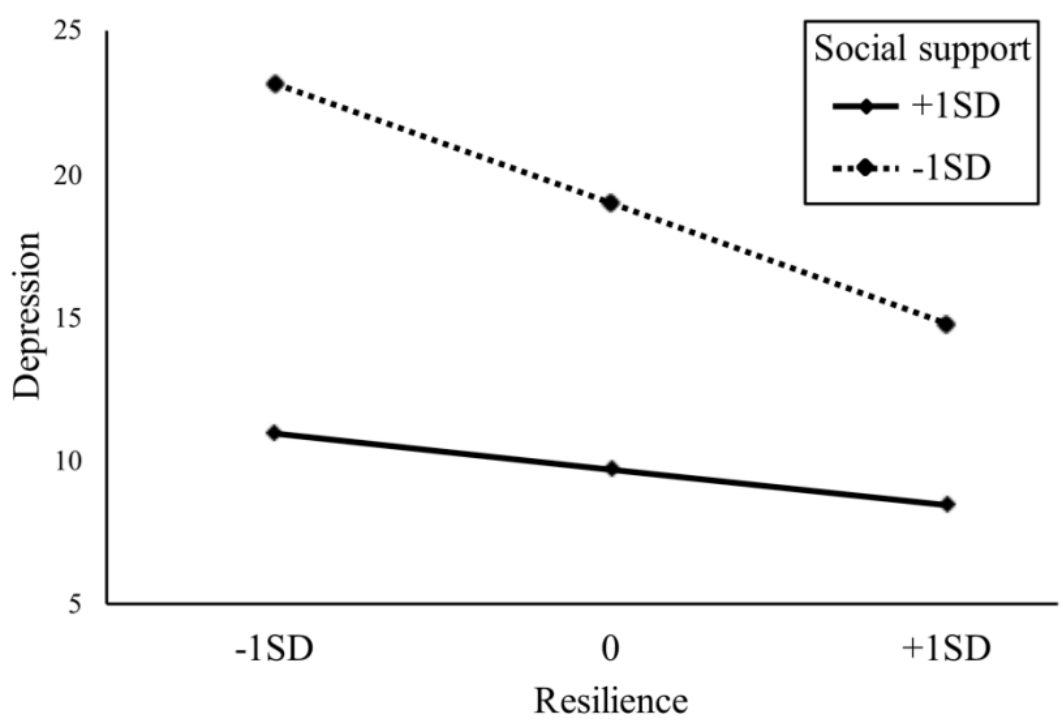

One component of the RSCA and one dimension of the MSPSS are both labeled

family support. Though the items in these two subscales differed, we tested the correlation between these components. They were highly correlated, $r(257)=.74, p<.001$. To ensure that our model was not a statistical artifact, we re-calculated the scores of resilience during the pandemic-related quarantine without the family support component and reran the moderated mediation model. The moderation of social support on the relationship between resilience and depression remained, $B=.01, S E=.004, p=.001,95 \% \mathrm{CI}=[.01, .02]$. See Table S3 for the adjusted results.

\section{Discussion}

To our knowledge, this is the first longitudinal analysis of adolescents' resilience before and during a pandemic-related quarantine. Previous research has shown that resilience can improve via interventions (e.g., Hodder et al. 2011; Tam et al. 2020), however, it was 
previously unknown what circumstances might decrease resilience. During the quarantine, the adolescents in our sample were under mandated social distancing and were attending school from home. Resiliency had strong internal reliability at both time points. However, adolescents' scores were only moderately correlated with their scores before the pandemic. Further, there was a significant overall drop in scores from pre-pandemic to during the pandemic-related quarantine. These results suggest that neither individual differences nor the strength of resilience were stable under the influence of the pandemic-related quarantine. This might not be surprising given that the developers of the RSCA assumed the measure quantified coping processes rather than trait strength (Hu and $\mathrm{Gan} 2008)$. The finding that resilience was negatively associated with depressive symptoms and sleep problems supports the protective model of resilience (Rutter 1987). The mediating effect of depression also aligns with the assumption that the depressive symptoms might be a precursor to the onset of sleep problems (Patten et al. 2000). The fact that the pattern remained when using an earlier pre-pandemic measure of resilience, suggests that, though resilience was influenced by pandemic-related quarantine, it can still predict future coping processes.

Our findings indicated that social support moderates the effect of resilience on depression. This finding is consistent with our hypothesis and supports the buffering model of social support (Cohen and Wills 1985). Specifically, for those adolescents with low social support, resilience was negatively related to depressive symptoms, whereas for those with high social support, the depressive symptoms generally remained low regardless of the adolescent's level of resilience. According to the buffering model, social support provides 
mental resources for adolescents to cope with depression, and personal resilience is largely unnecessary when enough social support is offered. Besides investigating and confirming the moderating effect of social support on the relationship between resilience and negative outcomes, we investigated the complementary relationship between resilience as an internal mental resource and social support as an external mental resource. Our results suggested even though the resilience might be weakened by the pandemic or the related quarantine, external mental resources from friends, families and significant others may compensate the loss of resilience.

Several limitations must be acknowledged. First, sampling bias may exist because the adolescents were not randomly selected, but were a convenience sample. Second, though this study showed the moderating effects of social support, the effect size was relatively small, therefore, future studies should further examine the moderating effect of social support on the association between resilience and depression.

Third, the associations among resilience, depression, social support, and sleep problems were found from a cross-sectional dataset, so the findings are not indicative of causality. Indeed, the mediation model could have been examined with the factors in different positions, such as sleep problems as a mediator between resilience and depression rather than depression mediating the relationship between resilience and sleep problems. Previous research has shown both that depressive symptoms predict sleep problems (e.g., Patten et al. 2000) and that sleep problems predict depressive symptoms (e.g., Breslau et al. 1996). It is thus likely there is a bi-directional relationship. Fourth, this study did not take other socio- 
demographic characteristics (e.g., socioeconomic status) into account, in part because the sample was fairly homogenous. All adolescents were from similar middle-class neighborhoods in China, and so the generalizability of the conclusions should be examined further in the future.

Despite these limitations, this study contributes new knowledge regarding the influence of pandemic-related quarantine on adolescents' resilience and the relationships between resilience, depression, sleep problems, and social support. In particular, we found evidence that the pandemic or pandemic-related quarantine reduced the resilience of adolescents. Given that we selected a sample from a city with limited confirmed cases and no pandemic-related deaths but strict lockdown administrative orders, we eliminated the negative physical effects from the pandemic. This more strongly suggests that our findings are due to psychological factors from the social isolation during the pandemic-related quarantine, rather than illness or immediate concerns for one's health.

Our findings have potential implications for adolescents in future pandemics or second waves of the current pandemic as well as related quarantines. More broadly, our findings might also have implications for any adolescent in social isolation. Our results suggest that adolescents without stable coping processes, especially those without social support, are the most likely to suffer during social isolation. Efforts to identify such adolescents could lead to targeted therapy or resilience training to improve adolescents' mental health outcomes during times of social isolation. 


\section{Declarations}

\section{Ethics approval}

The study was approved by the Institutional Review Board at Department of Psychology of Tsinghua University.

\section{Informed Consent}

Informed consent was obtained from all individual participants and their parents in the study.

\section{Conflicts of interest}

On behalf of all authors, the corresponding author states that there is no conflict of interest. 


\section{References}

Breslau, N., Roth, T., Rosenthal, L., \& Andreski, P. (1996). Sleep disturbance and psychiatric disorders: A longitudinal epidemiological study of young adults. Biological Psychiatry, 39(6), 411-418. https://doi.org/10.1016/0006-3223(95)00188-3

Brooks, S. K., Webster, R. K., Smith, L. E., Woodland, L., Wessely, S., Greenberg, N., \& Rubin, G. J. (2020). The psychological impact of quarantine and how to reduce it: rapid review of the evidence. The Lancet, 395(10227), 912-920. https://doi.org/10.1016/S0140-6736(20)30460-8

Buysse, D. J., Reynolds, C. F., Monk, T. H., Berman, S. R., \& Kupfer, D. J. (1989). The Pittsburgh sleep quality index: A new instrument for psychiatric practice and research. Psychiatry Research, 28(2), 193-213. https://doi.org/10.1016/0165-1781(89)90047-4

Caballero-Domínguez, C. C., Jiménez-Villamizar, M. P., \& Campo-Arias, A. (2020). Suicide risk during the lockdown due to coronavirus disease (COVID-19) in Colombia. Death Studies, O(0), 1-6. https://doi.org/10.1080/07481187.2020.1784312

Chatburn, A., Coussens, S., \& Kohler, M. J. (2014). Resiliency as a mediator of the impact of sleep on child and adolescent behavior. Nature and Science of Sleep, 6, 1-9. https://doi.org/10.2147/NSS.S54913

Cohen, S., \& Wills, T. A. (1985). Stress, social support, and the buffering hypothesis. Psychological Bulletin, 98(2), 310-357. https://doi.org/10.1037/0033-2909.98.2.310

Fendrich, M., Weissman, M. M., \& Warner, V. (1990). Screening for depressive disorder in children and adolescents: Validating the center for epidemiologic studees depression 
scale for children. American Journal of Epidemiology, 131(3), 538-551.

https://doi.org/10.1093/oxfordjournals.aje.a115529

Ford, D. E., \& Kamerow, D. B. (1989). Epidemiologic Study of Sleep Disturbances and Psychiatric Disorders: An Opportunity for Prevention? JAMA: The Journal of the American Medical Association, 262(11), 1479-1484.

https://doi.org/10.1001/jama.1989.03430110069030

Hall-Lande, J. A., Eisenberg, M. E., Christenson, S. L., \& Neumark-Sztainer, D. (2007). Social isolation, psychological health, and protective factors in adolescence. Adolescence, 42(166), 265-286.

Hayes, A. (2013). Integrating mediation and moderation analysis: Fundamentals using PROCESS. In Introduction to Mediation, Moderation and Conditional Process Analysis. https://doi.org/978-1-60918-230-4

Hébert, M., Lavoie, F., \& Blais, M. (2014). Síndrome do Estresse Pós-Traumático/PTSD em adolescentes vítimas de violência sexual: A resiliência e o suporte social como fatores de proteção. Ciencia e Saude Coletiva, 19(3), 685-694. https://doi.org/10.1590/141381232014193.15972013

Hjemdal, O., Vogel, P. A., Solem, S., Hagen, K., \& Stiles, T. C. (2011). The relationship between resilience and levels of anxiety, depression, and obsessive-compulsive symptoms in adolescents. Clinical Psychology and Psychotherapy, 18(4), 314-321. https://doi.org/10.1002/cpp.719

Hodder, R. K., Daly, J., Freund, M., Bowman, J., Hazell, T., \& Wiggers, J. (2011). A school- 
based resilience intervention to decrease tobacco, alcohol and marijuana use in high school students. BMC Public Health, 11. https://doi.org/10.1186/1471-2458-11-722

Hu, Y., \& Gan, Y. (2008). Development and psychometric validity of the resilience scale for chinese adolescents. Acta Psychologica Sinica, 40(8), 902-912. https://doi.org/10.3724/SP.J.1041.2008.00902

JASP Team. (2019). JASP (version 0.11.1) [Computer software]. Retrieved from https://jaspstats.org/download/

Jeong, H., Yim, H. W., Song, Y. J., Ki, M., Min, J. A., Cho, J., \& Chae, J. H. (2016). Mental health status of people isolated due to Middle East Respiratory Syndrome. Epidemiology and Health, 38, e2016048. https://doi.org/10.4178/epih.e2016048

Kaynak, Ö., Lepore, S. J., \& Kliewer, W. L. (2011). Social support and social constraints moderate the relation between community violence exposure and depressive symptoms in an urban adolescent sample. Journal of Social and Clinical Psychology, 30(3), 250269. https://doi.org/10.1521/jscp.2011.30.3.250

Levesque, R. J. R. (2011). Resilience. In R. J. R. Levesque (Ed.), Encyclopedia of Adolescence (pp. 2369-2373). New York, NY: Springer.

Li, S., Wang, Y., Xue, J., Zhao, N., \& Zhu, T. (2020). The impact of covid-19 epidemic declaration on psychological consequences: A study on active weibo users. International Journal of Environmental Research and Public Health, 17(6). https://doi.org/10.3390/ijerph17062032

Liang, L., Ren, H., Cao, R., Hu, Y., Qin, Z., Li, C., \& Mei, S. (2020). The effect of COVID- 
19 on youth mental health. Psychiatric Quarterly, (1163), 1-12.

https://doi.org/10.1007/s11126-020-09744-3

Mota, C. P., \& Matos, P. M. (2015). Adolescents in institutional care: Significant adults, resilience and well-Being. Child and Youth Care Forum, 44(2), 209-224. https://doi.org/10.1007/s10566-014-9278-6

Murberg, T. A., \& Bru, E. (2004). Social support, negative life events and emotional problems among Norwegian adolescents. School Psychology International, 25(4), 387402. https://doi.org/10.1177/0143034304048775

Patten, C. A., Choi, W. S., Gillin, J. C., \& Pierce, J. P. (2000). Depressive symptoms and cigarette smoking predict development and persistence of sleep problems in US adolescents. Pediatrics, 106(2), e23--e23. https://doi.org/10.1542/peds.106.2.e23

Pössel, P., Burton, S. M., Cauley, B., Sawyer, M. G., Spence, S. H., \& Sheffield, J. (2018). Associations between Social Support from Family, Friends, and Teachers and depressive Symptoms in Adolescents. Journal of Youth and Adolescence, 47(2), 398412. https://doi.org/10.1007/s10964-017-0712-6

Quinton, D., Pickles, A., Maughan, B., \& Rutter, M. (1993). Partners, peers, and pathways: Assortative pairing and continuities in conduct disorder. Development and Psychopathology, 5(4), 763-783. https://doi.org/10.1017/S0954579400006271

Rutter, M. (1987). Psychosocial resilience and protective mechanisms. American Journal of Orthopsychiatry, 57(3), 316-331. https://doi.org/10.1111/j.1939-0025.1987.tb03541.x Sprang, G., \& Silman, M. (2013). Posttraumatic stress disorder in parents and youth after 
health-related disasters. Disaster Medicine and Public Health Preparedness, 7(1), 105110. https://doi.org/10.1017/dmp.2013.22

Tam, C. C., Li, X., Benotsch, E. G., \& Lin, D. (2020). A Resilience-Based Intervention Programme to Enhance Psychological Well-Being and Protective Factors for Rural-toUrban Migrant Children in China. Applied Psychology: Health and Well-Being, 12(1), 53-76. https://doi.org/10.1111/aphw.12173

van Schalkwijk, F. J., Blessinga, A. N., Willemen, A. M., Van Der Werf, Y. D., \& Schuengel, C. (2015). Social support moderates the effects of stress on sleep in adolescents. Journal of Sleep Research, 24(4), 407-413. https://doi.org/10.1111/jsr.12298

William Li, H. C., Chung, O. K. J., \& Ho, K. Y. (2010). Center for epidemiologic studies depression scale for children: Psychometric testing of the Chinese version. Journal of Advanced Nursing, 66(11), 2582-2591. https://doi.org/10.1111/j.13652648.2010.05440.x

Ye, Z., Chen, L., Harrison, S. E., Guo, H., Li, X., \& Lin, D. (2016). Peer victimization and depressive symptoms among rural-to-urban migrant children in China: The protective role of resilience. Frontiers in Psychology, 7(OCT), 1-10. https://doi.org/10.3389/fpsyg.2016.01542

Ying, L., Wu, X., Lin, C., \& Jiang, L. (2014). Traumatic severity and trait resilience as predictors of posttraumatic stress disorder and depressive symptoms among adolescent survivors of the Wenchuan earthquake. PLoS ONE, 9(2). 
https://doi.org/10.1371/journal.pone.0089401

Zhao, J., Song, F., Chen, Q., Li, M., Wang, Y., \& Kong, F. (2018). Linking shyness to loneliness in Chinese adolescents: The mediating role of core self-evaluation and social support. Personality and Individual Differences, 125, 140-144. https://doi.org/10.1016/j.paid.2018.01.007

Zhou, S., Wang, L., Yang, R., Yang, X., Zhang, L., Guo, Z., Chen, J., Wang, J., \& Chen, J. (2020). Sleep problems among Chinese adolescents and young adults during the coronavirus-2019 pandemic. Sleep Medicine. https://doi.org/https://doi.org/10.1016/j.sleep.2020.06.001

Zhou, X., Wu, X., \& An, Y. (2016). Understanding the Relationship between Trauma Exposure and Depression among Adolescents after Earthquake: The Roles of Fear and Resilience. Frontiers in Psychology, Vol. 7, p. 2044. Retrieved from https://www.frontiersin.org/article/10.3389/fpsyg.2016.02044

Ziaian, T., de Anstiss, H., Antoniou, G., Baghurst, P., \& Sawyer, M. (2012). Resilience and its association with depression, emotional and behavioural problems, and mental health service utilisation among refugee adolescents living in south Australia. International Journal of Population Research, 2012, 1-9. https://doi.org/10.1155/2012/485956

Zimet, G. D., Dahlem, N. W., Zimet, S. G., \& Farley, G. K. (1988). The multidimensional scale of perceived social support. Journal of Personality Assessment, 52(1), 30-41. https://doi.org/10.1207/s15327752jpa5201_2

Zolkoski, S. M., \& Bullock, L. M. (2012). Resilience in children and youth: A review. 
Children and Youth Services Review, 34(12), 2295-2303.

https://doi.org/10.1016/j.childyouth.2012.08.009 